Psychopharmacologia (Berl.) 23, 319-333 (1972)

(C) by Springer-Verlag 1972

\title{
Central Effects of Atropine upon Aversive Classical Conditioning in Rabbits*
}

\author{
D. Downs*, C. Cardozo, N. Schnemderman, A. L. Yehle**, \\ D. H. VANDERCAR ***, and G. ZWHLING \\ Department of Psychology and Laboratory for Quantitative Biology, University of \\ Miami, Coral Gables, Florida 33124, U.S.A.
}

Received March 8, 1971 ; Final Version September 10, 1971

\begin{abstract}
Rabbits were given classical discrimination conditioning with one of two tones followed by shock. In Experiment I, 40 rabbits were trained under saline, 10,18 or $26 \mathrm{mg} / \mathrm{kg}$ atropine sulfate or $18 \mathrm{mg} / \mathrm{kg}$ methylatropine. Six rabbits in Experiment 2 were conditioned, then given further sessions with saline, and 18, 26 and $34 \mathrm{mg} / \mathrm{kg}$ atropine sulfate and methylatropine. In Experiment 3,18 rabbits were conditioned and then given two extinction sessions under saline or $34 \mathrm{mg} / \mathrm{kg}$ atropine sulfate or methylatropine followed by extinction under saline. Chief findings were (a) atropine sulfate but not methylatropine disrupted acquisition and maintenance of conditioned eyeblinks, (b) neither drug affected unconditioned blinks, (c) fewer blinks occurred in extinction under atropine sulfate than under methylatropine or saline, (d) rabbits extinguished under atropine sulphate showed higher percentages of eyeblinks when tested without drug. Disruptions in performance of learned eyeblink responses appeared to be due to drug interference with central cholinergic transmission.
\end{abstract}

Key words: Atropine Sulfate - Atropine Methylnitrate - Extinction Aversive Classical Conditioning - Rabbits - Differential Conditioning.

The present study assessed the central effects of atropine upon the acquisition, maintenance and extinction of classically conditioned discriminations between tones. In order to separate the central from peripheral effects of atropine, two forms of the drug were used. One of these, atropine sulfate (AS) is the tertiary form of the drug and passes readily through the blood brain barrier. The second form of the drug,

* Supported by grant GB-7944 and GB-24713 from the National Science Foundation and by NICHHD training grant 00187 in Quantitative Organismic Biology to the Laboratory for Quantitative Biology. We thank Stasenburgh Laboratories, division of Wallace and Tiernan, Inc. Rochester, New York for kindly supplying the atropine methylnitrate (Metropine).

* Now at the University of Michigan.

** NIMH postdoctoral fellow. Now at Memphis State University.

*** NASA predoctoral fellow. Now at Rockefeller University. 
atropine methylnitrate (MA), is a quaternary ammonium derivative, which exerts the same peripheral action without readily passing through the barrier (Carlton, 1962; Giarman and Pepeu, 1964; Khavari and Maickel, 1967).

The decremental effects of centrally acting antimuscarinic drugs on the acquisition of learned responses has been previously demonstrated (Bohdanecky et al., 1967; Buresova et al., 1964; Khavari and Maickel, 1967) although at least one study (Meyers et al., 1964) reported that scopolamine did not disrupt the retention of a conditioned avoidance response.

Although antimuscarinic drugs such as atropine and scopolamine have almost uniformly led to decrements in learned performance, several different explanations have been offered to account for the results. Carlton (1963), for example, suggested that these drugs cause a performance decrement by disrupting the normal process of habituation. Meyers (1965) proposed that antimuscarinic drugs may interfere with memory consolidation. Overton (1966) offered a stimulus change explanation emphasizing differences in the chemical state of the organism in the training and test situations. Deutsch and his collaborators (Deutsch, 1969; Deutsch et al., 1966a; Deutsch et al., 1966 b) have presented evidence that the performance of learned tasks is dependent upon the functioning of central cholinergic synapses.

Since behavioral experiments with antimuscarinic drugs have used widely discrepant procedures, it often has been difficult to make meaningful comparisons among hypotheses. In the present study the performance of rabbits given aversive classical conditioning was examined as a function of atropine administration during original acquisition, retention training, or extinction.

\section{Methods \\ Experiment I: Acquisition}

Subjects. The animals were 40 experimentally naive male albino rabbits (Oryctolagus cuniculus) about 90 days old and weighing approximately $2.3 \mathrm{~kg}$.

Apparatus and Preparation. The conditioning apparatus and manner in which each rabbit was restrained have been described previously (Gormezano, 1966). During experimental sessions each animal was restrained in a Plexiglas box with gross body and head movements further restricted by clamping the ears to the front plate of a stock with a clamp covered with foam rubber.

A day prior to being placed in the experimental situation each rabbit was anesthetized with sodium pentobarbital $(25-35 \mathrm{mg} / \mathrm{kg}, \mathrm{i.v}$.$) , and$ an incision was made in the skin exposing a portion of the skull above 
the right motor cortex. Two burr holes were bored through the skull and bipolar, silver, epidural screw electrodes were implanted in the bone and fixed with dental cement in order to record electrocorticographic activity. Two steel safety pins inserted chronically into the skin of the rabbit's right front leg and left rear haunch were used to record heart rate (HR). A small nylon loop was sutured through the epithelium layer of the nictitating membrane (NM) to permit recording of NM responses. Neither the HR safety pins nor the loop sutured through the NM induced observable discomfort, attempts to dislodge the pins or infection during the course of the experiment.

During each session the outer lids of the animal's right eye were held open by small steel clips, which also served as electrodes for electric shock which was the unconditioned stimulus. The NM loop was then mechanical ly coupled with a small thread to a plastic arm on which was exposed a Budd type F20 "Flexagage" consisting of two strain gauges mounted back to back on a plastic strip (Yehle, 1968). Extensions of the NM deformed the "Flexagage", the output of which was passed through a demodulator and amplified on a Grass Model III-A EEG machine. Unlike the cat, the NM response in the rabbit is essentially somatic. Lierse (1960) has described the presence of striated muscle attached to the NM, and we have observed that neuromuscular blocking agents such as Flaxedil readily prevent the occurrence of NM responses.

The conditioned stimuli (CSs) were two tones of 550 and $3400 \mathrm{~Hz}$, delivered by Ameco code practice oscillators at an intensity of $78 \mathrm{db}$ SPL. The unconditioned stimulus was a 0.5 sec duration, 3 ma. a.c. shock delivered by a Grason-Stadler shock generator.

Procedure. The rabbits were randomly assigned to one of five groups of eight animals each. During acquisition, each of these groups received daily subcutaneous injections of either saline or 10,18 or $26 \mathrm{mg} / \mathrm{kg}$ of $\mathrm{AS}$ or $18 \mathrm{mg} / \mathrm{kg} \mathrm{MA}$. Injections were given $10 \mathrm{~min}$ before the beginning of each session. The reason that the rabbits received dosages in excess of those normally used with most other mammals including man is that they possess a special enzyme, atropinesterase, that destroys belladonna alkaloids (Innes and Nickerson, 1966; Stormont and Suzuki, 1970). Dosages as large as $200 \mathrm{mg} / \mathrm{kg}$, however, have been used in learning experiments conducted upon rats (Overton, 1966).

Each group received one day of adaptation to tones without shock and nine days of acquisition training. Ten minutes preceding the adaptation session each rabbit was injected with $3 \mathrm{ml}$ of isotonic saline. During adaptation and acquisition sessions each group received 36 presentations of each tone with the restriction that no more than two of each frequency CS would occur consecutively. During acquisition, the subjects received classical discrimination conditioning in which one of the tones (designated 
$\mathrm{CS}^{+}$) was immediately followed by shock, whereas the other tone (designated $\mathrm{CS}^{-}$) was not. One-half of each group received shock in conjunction with each frequency tone. The CS duration and interstimulus intervals were $0.75 \mathrm{sec}$ and the intertrial interval was 90 sec.

Of the 72 trials per day in acquisition, 32 presentations of the $\mathrm{CS}^{+}$ were paired with shock and four presentations (trials 17, 36, 53, 72) were presented unpaired as test trials. Of the $36 \mathrm{CS}^{-}$trials, four presentations (trials $\mathbf{1 8}, \mathbf{3 5}, \mathbf{5 4}, \mathbf{7 1}$ ) served as test trials.

Response Measurements and Statistical Analyses. On each adaptation and acquisition trial the first NM extention resulting in at least a $1.5 \mathrm{~mm}$ pen ( $0.5 \mathrm{~mm}$ membrane) deflection from baseline and occurring from 0 to $0.75 \mathrm{sec}$ after CS onset was recorded as a conditioned response (CR). The HR changes were measured only on test trials and were recorded both as post-minus pre-CS onset difference scores and as percent changes from baseline. In each case this was accomplished by comparing the temporal duration of the 10 heart beats before CS onset with the temporal duration of the first 10 beats following CS onset. Changes in electrocorticographic activity were evaluated by two independent observers who were not familiar with the design of the experiment. During each baseline period preceding a test trial as well as during the CS presentation the observers determined whether the cortical activity consisted of slow (2-4 per sec), synchronous high amplitude delta waves or whether it consisted of desynchronized, low voltage activity. Inter-observer agreement was $93 \%$. A significance level of $p<0.05$ was used in the statistical analyses of all three experiments.

\section{Experiment 2: Maintenance of Conditioning}

Six experimentally naive male New Zealand albino rabbits were used. The basic apparatus was the same as in Experiment I. Instead of the strain gauge used to record NM activity in Experiment I however, a corneo-retinal transducer was used to provide a frequency measure of eyeblink activity. Except for artifacts associated with transduction, corneo-retinal potentials, NM extensions, eyeblinks and retraction of the eyeball into the orbit, form a unitary response constellation in the rabbit. Using our procedures, frequency of NM responses, eyeblinks and corneoretinal potentials are perfectly correlated with one another (VanDercar et al., 1969).

Stimulation parameters were kept identical with Experiment I. Each subject, however, received two days of adaptation training followed by a variable number of acquisition sessions until criteria of more than $90 \%$ eyeblink responding to the $\mathrm{CS}^{+}$and less than $25 \%$ eyeblink responding to the CS- were met for three successive sessions. Once this baseline 
was established each animal had to meet the criteria for two successive sessions under saline before receiving each administration of atropine. In view of the findings of Buresova et al. (1964) that the antimuscarinic disruption of behavior is reduced by overtraining, higher dosages of AS and MA were used that in the first experiment. Thus during each drug session the subject received either 18,26 or $34 \mathrm{mg} / \mathrm{kg}$ of AS or MA. Five of the six animals received two complete series comprising all of the different dosage levels of AS and MA delivered in a random sequence. The sixth animal received only one complete drug series because of subsequent difficulties in maintaining stable baseline behavior.

\section{Experiment 3: Extinction}

The subjects were 18 naive male New Zealand rabbits. The apparatus and acquisition training procedures were identical with those of Experiment 2 except that all rabbits received exactly two days of adaptation and 12 days of acquisition training. After acquisition training, subjects were assigned to saline, MA or AS conditions with six rabbits in each group. Extinction training (with tones but no shocks) was given for two days under $3 \mathrm{ml}$ saline or $34 \mathrm{mg} / \mathrm{kg}$ AS or MA. This was followed by a third extinction day in which all subjects were injected with $3 \mathrm{ml}$ of saline.

\section{Results}

\section{Experiment I: Acquisition}

Mean daily percentages of NM responses to the $\mathrm{CS}^{+}$and $\mathrm{CS}^{-}$are shown for each group during acquisition in Fig. 1. It can be seen that at each dosage AS retarded the rate of CRs to the $\mathrm{CS}^{+}$in relation to the control group. In contrast, at the $18 \mathrm{mg} / \mathrm{kg}$ dosage the MA group's acquisition curve resembled that of the control group. An analysis of variance in conjunction with Duncan range post-tests was conducted upon $\mathrm{NM} \mathrm{CR}^{+}$responses cumulated over acquisition sessions. These analyses confirmed that all of the AS groups differed reliably from the MA and saline control groups. In contrast, the latter two groups did not differ significantly from each other.

Although AS decreased the rate of acquisition of NM responses to the $\mathrm{CS}^{+-}$the drug did not influence the frequency of NM unconditioned responses. These unconditioned responses (not shown in Fig. 1) approached a frequency of $100 \%$ in all groups throughout acquisition. Mean latencies and response amplitudes of the unconditioned responses did not differ from one another and analyses of variance conducted on these measures among groups did not approach significance.

Percentages of baseline electrocorticographic synchronization varied from approximately $30 \%$ delta activity in the saline and MA groups to 


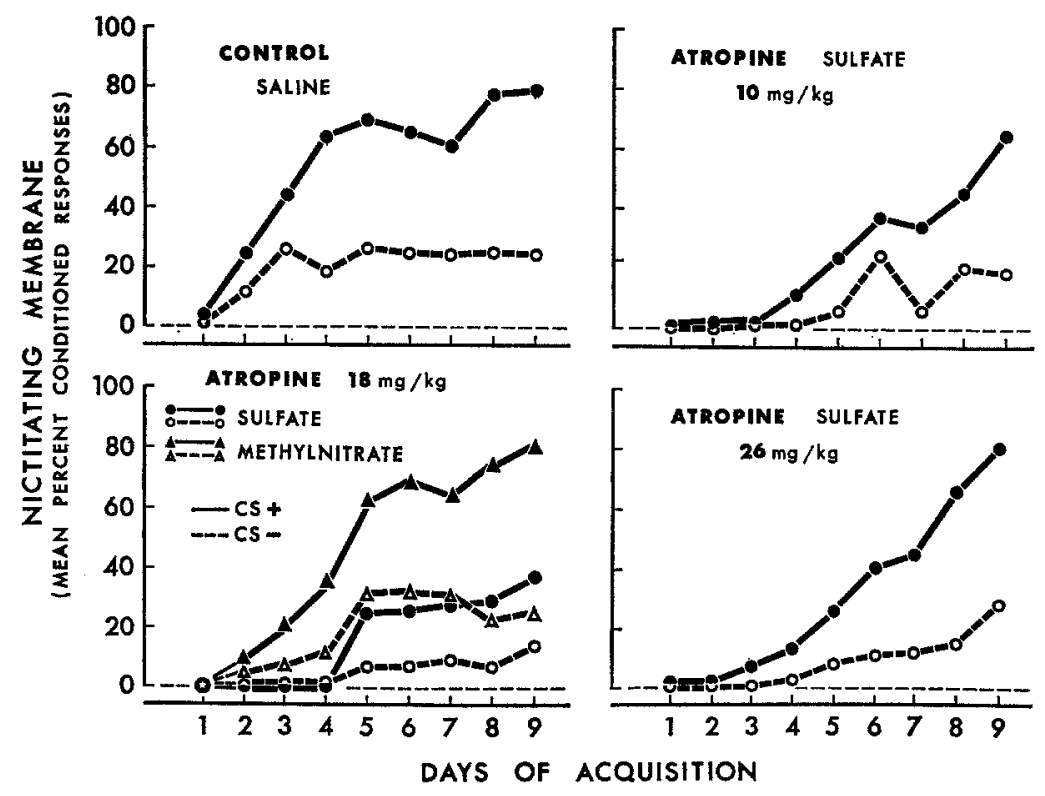

Fig. 1. Mean daily percentages of membrane responses to the CS+ and CS- for each saline or atropine group during acquisition

above $90 \%$ delta activity in the $26 \mathrm{mg} / \mathrm{kg}$ AS group. An analysis of variance in conjunction with Duncan range tests was conducted upon percentages of baseline synchronization cumulated over sessions. These analyses indicated that reliable differences existed only between the AS groups vs. the MA and saline control groups. An orthogonal polynomial trend analysis for unequally spaced intervals was conducted upon the cumulative means of the control and AS groups. This analysis indicated a significant linear trend signifying a dose-dependent relationship.

In order to test the equivalence of MA and AS upon peripheral responding, baseline $H R$ and conditioned $H R$ changes were examined. These measures were also used to assess the within-session (time-response) effects of the drugs. Mean baseline HR before daily injection was approximately 210 beats per min (BPM) throughout acquisition. After injection mean HR in the saline control group decreased slightly during the course of each session. In contrast, all groups injected with either MA or AS showed a pronounced increase in HR. Mean HR in the atropine groups was $263 \mathrm{BPM}$ at the outset of each session and decreased gradually to 243 BPM just before the last daily trial. An analysis of variance conducted upon mean baseline HR just prior to each daily injection revealed no 


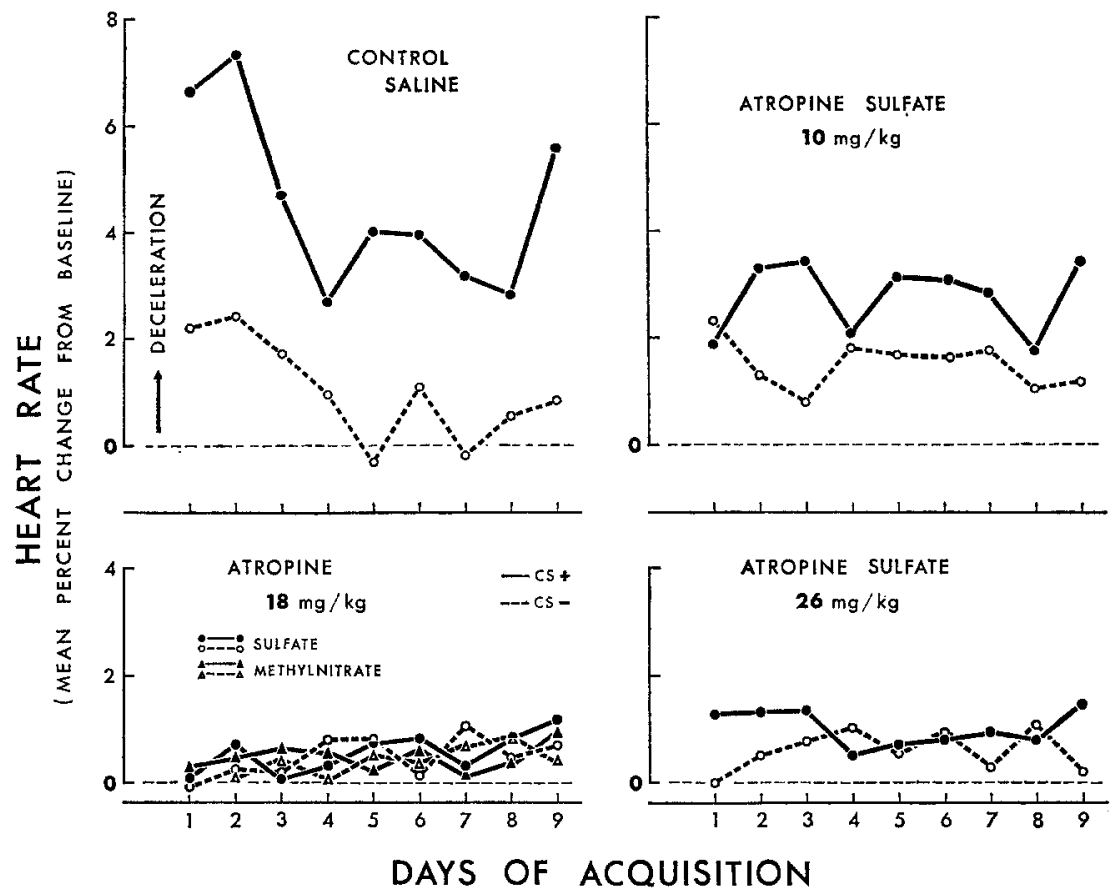

Fig. 2. Mean percent heart rate changes from baseline to $\mathrm{CS}^{+}$and $\mathrm{CS}^{-}$test trials for each saline or drug group during acquisition

significant differences among groups. In contrast, an HR baseline measure (mean HR baseline before each CS+ test trial) cumulated over acquisition sessions indicated the presence of reliable differences among groups. Duncan range tests indicated that significant differences existed only between the control group and each of the drug groups. The drug groups did not differ reliably from one another.

The mean daily $\mathrm{HR}$ responding to the $\mathrm{CS}^{+}$and $\mathrm{CS}^{-}$were analyzed separately in terms of (a) percent HR changes from baseline, and (b) difference scores, comparing durations of 10 heart beats before with 10 heart beats after CS onset. Fig. 2 presents the mean daily HR percent changes from baseline for each group. It can be seen that in contrast to the NM findings, the greatest $\mathrm{HR}$ responses to the $\mathrm{CS}^{+}$in the saline group occurred during the first two days of acquisition. Moreover, clear $\mathrm{HR}$ differentiation in this group was evident during each acquisition session.

Fig. 2 also suggests that the $10 \mathrm{mg} / \mathrm{kg}$ doses of AS and MA reduced but did not eliminate the HR response to the $\mathrm{CS}^{+}$. In contrast, the 
$18 \mathrm{mg} / \mathrm{kg}$ doses of AS and MA both abolished HR conditioning. Analyses of variance of $\mathrm{HR}, \mathrm{CRs}$ to the $\mathrm{CS}^{+}$cumulated over all days of acquisition revealed significant differences among groups using either percentage change from baseline or post-minus pre-CS onset differences. Similar analyses based upon discrimination ( $\mathrm{CR}^{+}$minus $\mathrm{CR}^{-}$) scores also revealed reliable differences. Duncan range tests conducted upon HR responses to the $\mathrm{CS}^{+}$as well as upon $\mathrm{HR}$ discrimination scores indicated that the 18 and $26 \mathrm{mg} / \mathrm{kg}$ atropine groups, but not the $10 \mathrm{mg} / \mathrm{kg}$ atropine group differed reliably from the saline control group.

In Fig. 2 the daily percentages of $\mathrm{HR}$ responses to the $\mathrm{CS}^{+}$and $\mathrm{CS}^{-}$ are presented with each day representing the mean of four test trials. When percentages of HR responses to the $\mathrm{CS}^{+}$were cumulated over days, but analyzed as a function of differences among test trials it was found that all atropine groups revealed virtually no conditioning on the first test trial of each acquisition session. In contrast the $10 \mathrm{mg} / \mathrm{kg} \mathrm{AS}$, but not the 18 or $26 \mathrm{mg} / \mathrm{kg}$ atropine groups, showed a gradual increase in HR percent changes between the first and fourth daily test trials. By the fourth test trial CR magnitude in the $10 \mathrm{mg} / \mathrm{kg}$ AS group was indistinguishable from the saline control group. An analysis of variance conducted upon HR percent changes for test trial 1 cumulated over acquisition sessions revealed no significant differences among atropine groups. In contrast an analysis of variance in conjunction with Duncan range tests conducted on test trial 4 revealed that the $10 \mathrm{mg} / \mathrm{kg}$ AS group differed reliably from the other atropine groups.

\section{Experiment 2: Maintenance of Conditioning}

The six rabbits in this experiment were first given extensive classical conditioning training without drug and then tested under different dosages of AS and MA. To reach the criteria of three successive days of at least $90 \% \mathrm{CRs}$ to the $\mathrm{CS}^{+}$and less than $25 \% \mathrm{CRs}$ to the $\mathrm{CS}^{-}$the six rabbits required $9-19$ acquisition sessions (mean $=16$ ). Five of the six subjects then showed little difficulty recapturing baseline after saline or drug administration sessions; the mean number of saline days between drug injections being 5 . The sixth rabbit, D-4, had difficulty recapturing baseline after the fifth administration of atropine and was discontinued from the experiment after the sixth drug injection.

Fig. 3 shows the performance of one rabbit, D-1, throughout the course of training. It can be seen that once injections were instituted, D-1 seldom responded at less than $90 \% \mathrm{CRs}_{\text {s }}$ to the $\mathrm{CS}^{+}$during saline days. Administration of all dosages of MA did not markedly disturb the baseline; whereas, all dosages of AS led to pronounced decreases in responding to the $\mathrm{CS}^{+}$and almost always depressed the $\mathrm{CR}^{-}$baseline to $0 \%$. The few instances in which response decrements occurred during 


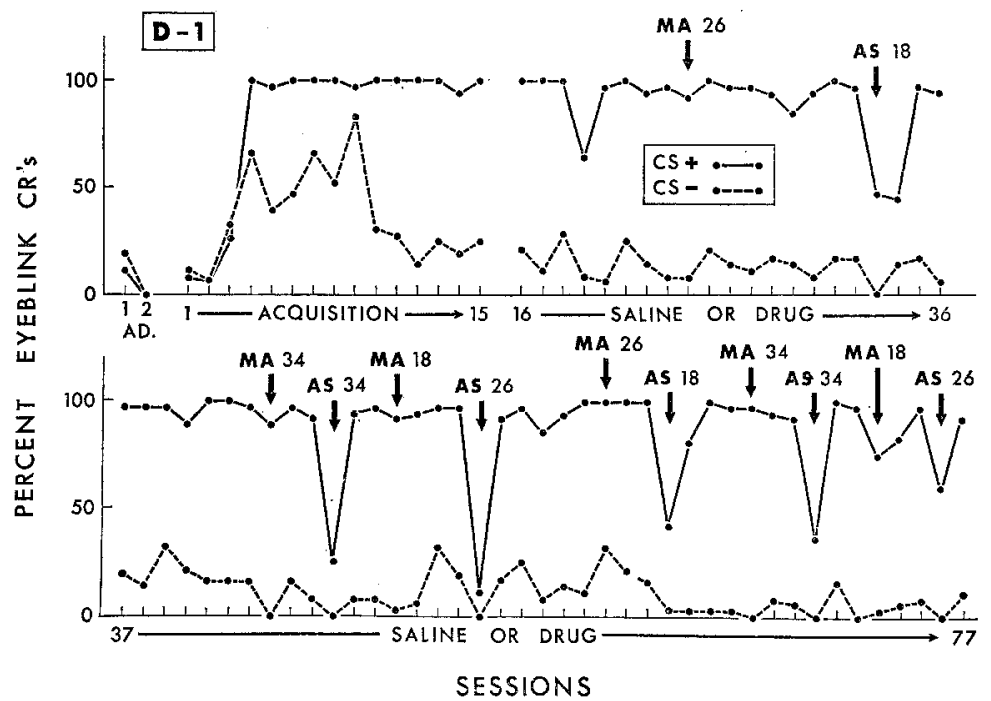

Fig. 3. Mean daily percentages of eyeblink responses to the $\mathrm{CS}^{+}$and $\mathrm{CS}^{-}$for rabbit, D-1, throughout each classical conditioning session. After the fifteenth acquisition session the rabbit received daily injections of either saline or 18,26 or $34 \mathrm{mg} / \mathrm{kg}$ of atropine sulfate $\left(A S^{\prime}\right)$ or methylatropine $(M A)$ as indicated

non-AS sessions were presumably without behavioral significance since they failed to occur consistently again at the same dosage, at other dosages or in other animals.

The mean daily level of eyeblink responding to the $\mathrm{CS}^{+}$after saline injections was $92 \%$. Mean responding after MA was $91 \%$. In contrast, mean frequency of eyeblink responses to the CS+ after AS injections was $46 \%$. Mean percentages of eyeblink responses to the $\mathrm{CS}^{+}$for each rabbit under saline and the different dosages of atropine are shown in Table 1. A two-way repeated measures analysis of variance conducted upon percentages of eyeblink CRs to the $\mathrm{CS}^{+}$at each dose of AS and MA confirmed a significant source of variation between responses to MA vs. AS but no significant dosage level or interaction effects.

As in the previous experiment both AS and MA rather uniformly increased baseline HR. A repeated measures analysis of variance comparing baseline HR under saline (sessions immediately preceding AS), AS and MA (both forms of atropine collapsed over dosage levels) revealed the presence of significant differences among treatments. Duncan range tests indicated reliable differences only between the saline vs. AS and MA conditions. 
Table 1. Mean percentages of eyeblink responses to the $C S^{+}$

\begin{tabular}{|c|c|c|c|c|c|c|c|}
\hline \multirow[t]{2}{*}{ Rabbit } & \multirow{2}{*}{$\frac{\text { Atropine }}{18 \mathrm{mg} / \mathrm{kg}}$} & \multicolumn{2}{|c|}{ Sulfate (AS) } & \multicolumn{3}{|c|}{ Methylatropine (MA) } & \multirow{2}{*}{$\begin{array}{l}\text { Saline } \\
3 \mathrm{ml}\end{array}$} \\
\hline & & $26 \mathrm{mg} / \mathrm{kg}$ & $34 \mathrm{mg} / \mathrm{kg}$ & $18 \mathrm{mg} / \mathrm{kg}$ & $26 \mathrm{mg} / \mathrm{kg}$ & $34 \mathrm{mg} / \mathrm{kg}$ & \\
\hline D-1 & 81 & 69 & 22 & 95 & 94 & 95 & 88 \\
\hline D-4 & 81 & 78 & 36 & 86 & 92 & 82 & 88 \\
\hline D-5 & 35 & 25 & 31 & 93 & 78 & 89 & 94 \\
\hline D-8 & 44 & 37 & 30 & 84 & 96 & 93 & 97 \\
\hline D-9 & 65 & 56 & 69 & 98 & 98 & 98 & 93 \\
\hline D-11 & 17 & 36 & 14 & 92 & 89 & 92 & 92 \\
\hline Mean & 54 & 50 & 34 & 91 & 91 & 92 & 92 \\
\hline
\end{tabular}

Five of the six rabbits in Experiment 2 revealed decelerative HR responses to the $\mathrm{CS}^{+}$. The sixth rabbit made greater magnitude responses to the $\mathrm{CS}^{+}$than to the $\mathrm{CS}^{-}$, but its $\mathrm{CS}^{+}$responses were clearly accelerative. Because accelerative as well as decelerative conditioning occurred in the present study, absolute values rather than algebraic summations were used in computing percent changes from baseline and post- minus pre-CS onset difference scores.

A repeated measures analysis of variance comparing HR changes to the $\mathrm{CS}^{+}$among saline (sessions immediately preceding AS days) AS and MA (with both forms of atropine collapsed over dosage levels) revealed significant differences. Duncan range test indicated that these differences were between the saline vs. AS and MA conditions. A repeated measures analysis comparing $\mathrm{HR}$ responses to the $\mathrm{CS}^{+}$at each dosage level of $\mathrm{AS}$ and MA failed to reveal reliable differences in drug or dosage effects.

\section{Experiment 3: Extinction}

Separate groups of rabbits in this experiment were extinguished under saline, AS or MA and then were given further extinction trials under saline. Fig. 4 depicts group mean eyeblink responses to the CSand $\mathrm{CS}^{-}$throughout adaptation, acquisition and extinction training. Analyses of variance conducted upon the eyeblink conditioning $\left(\mathrm{CR}^{+}\right)$ and discrimination $\left(\mathrm{CR}^{+}\right.$minus $\left.\mathrm{CR}^{-}\right)$scores during acquisition as shown in Fig. 4 revealed no significant differences among groups in conditioning or discrimination formation.

During extinction sessions all groups showed decreases in eyeblink CRs between the first and second sessions. Administration of AS in extinction appeared to depress eyeblink CRs relative to both MA and saline. Although the AS group made only $16 \%$ responses to the $\mathrm{CS}^{+}$ during the second extinction session under the drug, responding to the $\mathrm{CS}^{+}$was $94 \%$ during the drugless third extinction session. An analysis 


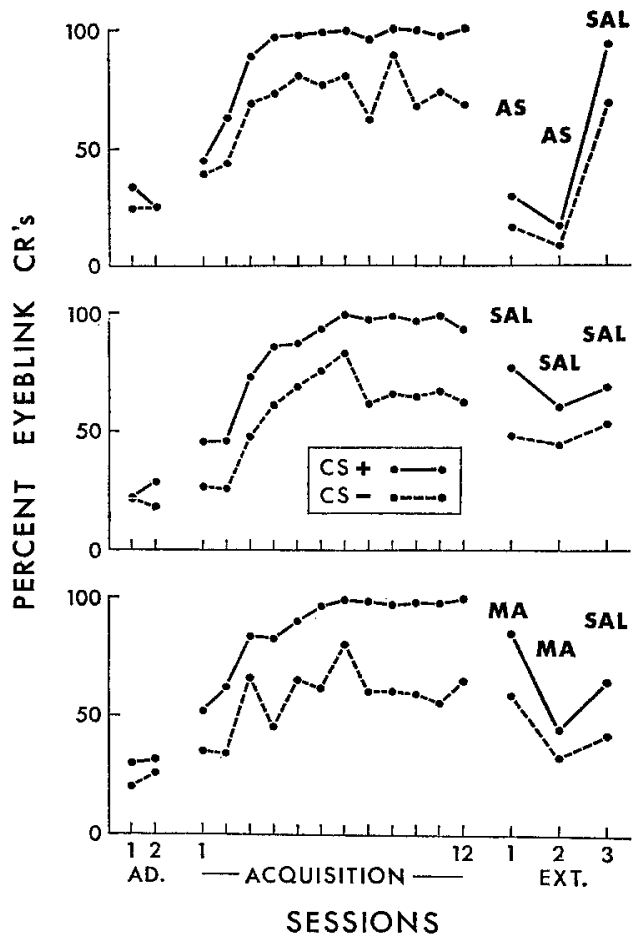

Fig. 4. Mean daily percentages of eyeblink responses to the $\mathrm{CS}^{+}$and $\mathrm{CS}-$ during adaptation $(A D)$, acquisition and extinction $(E X T)$ sessions. Extinction sessions in which rabbits in a group received atropine sulfate $(A S)$, methylatropine $(M A)$ or saline $(S A L)$ are noted

of variance conducted upon percent responses during the two drugged extinction sessions confirmed the presence of significant differences among groups. Duncan range post tests indicated that reliable differences occurred between the AS vs. saline and MA groups. A repeated measures analysis of variance comparing $\mathrm{CR}^{+}$performance among the three groups during the second and third extinction sessions failed to reveal significant differences as a function of drug treatment, but did reveal significant differences across days and showed a reliable interaction effect. Fig. 4 indicates that the significant interaction must have been due to the marked increase in responding between day 2 and day 3 in the AS group.

\section{Discussion}

The chief findings of the present study were (a) AS but not MA disrupted acquisition and maintenance of classically conditioned eyeblink or 
NM responses (b) neither drug influenced unconditioned NM responding, (c) AS but not MA increased delta activity in the electrocortigram, (d) AS depressed eyeblink responses to both $\mathrm{CS}^{+}$and $\mathrm{CS}^{-}$, (e) MA was at least as effective as AS in increasing baseline HR and depressing HR CRs, (f) eyeblink CRs during extinction occurred less frequently under AS than under MA or saline, and (g) rabbits showed very little eyeblink responding during extinction under AS, but revealed high levels of responding when later tested without the drug. It was also found that HR and eyeblink classical conditioning could be maintained for as long as 90 acquisition sessions and that classically conditioned eyeblink baselines were retrievable even after repeated drug administration.

Several alternative hypotheses have been offered to account for AS induced deficits on different learning tasks. These have been framed within the context of changes occurring in levels of motor activity or drive state, augmentation of previously inhibited responses to nonreinforced stimuli, state-dependency or direct influences upon the CNS.

The present findings of decreased responding under AS cannot be explained in terms of a general inhibition of motor activity. In studies in which centrally acting antimuscarinic drugs have been reported to influence spontaneous or overall motor functions the changes usually have been increases rather than decreases in motor activity (Harris, 1961 ; Meyers et al., 1964; Payne and Anderson, 1967; Pradhan and Roth, 1968). Furthermore, while AS blocks eyeblink CRs, Experiment I demonstrated that AS had no effect on the frequency, latency or magnitude of the unconditioned response. Since magnitudes of unconditioned responses have been reported to vary with changes in drive state (Martin, 1963; Razran, 1957), the absence of such changes in the present study suggests that CR decrements under AS were not due to reduced drive.

The observation that AS caused a decrease in responding to the CSas well as to the $\mathrm{CS}^{+}$indicates that the results of the present study cannot be explained in terms of an augmentation of previously inhibited responses to non-reinforced stimuli. It should be pointed out, however, that observations supporting the disinhibition hypothesis, were originally offered within the context of reductions in normal effects of nonreward within a free operant situation (Carlton, 1963).

The finding in Experiment I that AS retarded the acquisition of the NM response indicates that the disruption of conditioning by AS in the present study cannot be accounted for solely in terms of a state-dependence hypothesis. This argument is further strengthened by observations that none of the animals in Experiment 2 showed consistent improvements in performance under AS either within or across drug sessions. Possibly, however, if the AS animals in Experiment I which showed conditioning by Day 9 had been run under the drug for additional 
sessions and then tested under saline, response decrements under saline would have been observed.

The present observations that AS but not MA interfered with the acquisition and maintenance of eyeblink and NM CRs and that AS but not MA synchronized electrocorticographic activity agree with other evidence that MA does not readily pass through the blood-brain barrier (Carlton, 1962; Giarman and Pepeu, 1964; Khavari and Maickel, 1967). Observations that changes in HR baselines and HR conditioning were about the same under both drugs suggest that the peripheral effects of both agents were quite similar. The present findings thus suggest that an hypothesis stated in terms of CNS function is required to account for the data. Such theories have been proposed by Deutsch (1969) and by Buresova et al. (1964).

According to Deutsch, efficient performance of learned tasks is dependent upon the optimal functioning of central cholinergic synapses (Deutsch, 1969; Deutsch et al., 1966a; Deutsch et al., 1966 b). Interventions which interfere with optimal central cholinergic transmission may debilitate performance on some tasks. In the present experiments the cholinergic blocking agent AS, which passes through the blood brain barrier, disrupted eyeblink conditioning.

Since drugs which temporarily block acetylcholine transmission are transient in their effects and presumably do not influence the memory substrate itself, Deutsch (1969) has suggested that the effects upon behavior are also transitory. The finding in the second experiment that eyeblink conditioning baselines were immediately retrievable even on the first trial following drug days is consistent with Deutsch's hypothesis. In Experiment 2, AS interfered with the performance of the eyeblink CR. Since the CR was already well-learned it is unlikely that the response deficit in this specific situation was due to AS effects upon memory consolidation.

In Experiment 3 of the present study, AS but not MA depressed eyeblink responding relative to a saline control during the first two extinction sessions. When subjects previously given AS during extinction were given saline during a third extinction sessions their performance returned to the pre-extinction baseline. The results agree with the observations of Buresova et al. (1964) for the extinction of passive avoidance behavior in rats. These investigators suggested that AS blocks cholinergic pathways ordinarily used during learning. Consequently, they contended that extinction did not really occur under AS because the pathways involved in learned behavior were not functioning during drugged extinction.

Since some learning does occur under AS, Buresova et al. (1964) proposed that noncholinergic pathways mediated the learning. Experiment I 
of the present study confirmed Buresova's report that some learning can occur under AS, and Experiment 2 showed that AS disrupts but does not completely abolish a well-established eyeblink CR. Although Buresova et al. proposed a dual transmitter mechanism to account for such results, it is alternatively possible that systemically administered cholinergic blocking agents are never completely effective.

The three experiments in the present study each indicated that the effects of AS on classically conditioned eyeblink CRs were central rather than peripheral. Experiment 2 indicated that AS caused a deficit in the performance of learned responses rather than a learning deficit. Experiment 3 provided evidence that the pathways involved in the performance of learned responses are blocked in extinction by AS. All three experiments indicated decreased responding to the $\mathrm{CS}^{+}$under AS. Taken together, then, the present results suggest that at least one important effect of AS is to block the central cholinergic pathways responsible for the performance of learned responses.

\section{References}

Bohdanecky, Z., Jarvik, M. E., Carley, J. L.: Differential impairment of delayed matching in monkeys by scopolamine and scopolamine methylbromide. Psychopharmacologia (Berl.) 11, 293-299 (1967).

Buresova, O., Bures, J., Bohdanecky, Z., Weiss, T.: Effect of atropine on learning extinction, retention, and retrieval in rats. Psychopharmacologia (Berl.) 5, $255-263(1964)$.

Carlton, P. L.: Some behavioral effects of atropine and methyl atropine. Psychol. Rep. 10, 579-582 (1962).

- Cholinergic mechanisms in the control of behavior by the brain. Psychol. Rev. 70, $19-39$ (1963).

Deutsch, J. A.: The physiological basis of memory. Ann. Rev. Psychol. 20, 85-104 (1969).

- Hamburg, M. D., Dahl, H.: Anticholinesterase induced amnesia and its temporal aspects. Science 151, $221-223$ (1966).

- Leibowitz, S. F.: Amnesia or reversal of forgetting by anticholinesterase, depending simply on time of injection. Science 153, 1017 (1966).

Giarman, N. J., Pepeu, G.: The influence of centrally acting cholinolytic drugs on brain acetylcholine levels. Brit. J. Pharmacol. 23, 123-130 (1964).

Gormezano, I.: Classical conditioning. In: Experimental Methods and instrumentation in Psychology, pp. 385-420. J. B. Sidowski ed. New York: McGrawHill 1966.

Harris, L. S.: The effect of various anticholinergics on spontaneous activity of mice. Fed. Proc. 20, 395 (1961).

Innes, I. R., Nickerson, M.: Drugs inhibiting the action of acetylcholine on structures innervated by post-ganglionic parasympathetic nerves (antimuscarinic or atropinic drugs). In: The Pharmacological Basis of Therapeutics, pp. 521-545. L. S. Goodman and A. Gilman, eds. New York: MacMillan 1965.

Khavari, K. A., Maickel, R. P.: Atropine and atropine methyl bromide effects on behavior of rats. Int. J. of Neuropharmacol. 6, 301-306 (1967). 
Lierse, W.: Der Mechanismus der Nickhautbewegung des Kaninchens. Anat. Anz. $199,1-6(1960)$.

Martin, I.: A note on reflex sensitivity and formation of conditioned responses. Behav. Res. Ther. 1, 185-190 (1963).

Meyers, B.: Some effects of scopolamine on a passive avoidance response in rats. Psychopharmacologia (Berl.) 8, 111-119 (1965).

- Roberts, K. H., Riciputi, R. H., Domino, E. F.: Some effects of muscarinic cholinergic blocking drugs on behavior and the electrocorticogram. Psychopharmacologia (Berl.) 8, 289-300 (1964).

Overton, D. A.: State dependent learning produced by depressant and atropinelike drugs. Psychopharmacologia (Berl.) 10, 6-31 (1966).

Payne, R., Anderson, D. C.: Scopolamine-produced changes in activity and in the startle response: Implications for behavioral action. Psychopharmacologia (Berl.) 12, 83-90 (1967).

Pradhan, S. N., Roth, T.: Comparative behavioral effects of several anticholinergic agents in rats. Psychopharmacologia (Berl.) 12, 358-366 (1968).

Razran, G.: The dominance-contiguity theory of the acquisition of classical conditioning. Psychol. Bull. 54, 1-46 (1957).

Stormont, C., Suzuki, Y.: Atropinesterase and cocainesterase of rabbit serum: Localization of the enzyme activity in isozymes. Science 167, 200-202 (1970).

VanDercar, D. H., Swadlow, H. A., Elster, A., Schneiderman, N.: Nictitating membrane and corneo-retinal transducers for conditioning in rabbits. Amer. Psychol. 24, 262-264 (1969).

Yehle, A. L.: A method for transducing nictitating membrane and breathing rate responses in the rabbit. J. exp. Anal. Behav. 11, 207-208 (1968).

Dr. N. Schneiderman

Department of Psychology

University of Miami

Coral Gables, Florida 33124

U.S.A. 